\title{
Critical concepts, practice recommendations, and research perspectives of pixantrone therapy in non-Hodgkin lymphoma: a SIE, SIES, and GITMO consensus paper
}

Pier Luigi Zinzani ${ }^{1}$, Paolo Corradini ${ }^{2}$, Maurizio Martelli ${ }^{3}$, Giorgio Minotti ${ }^{4}$, Stefano Oliva ${ }^{5}$, Michele Spina ${ }^{6}$, Giovanni Barosi ${ }^{7}$, Sante Tura ${ }^{8}$

${ }^{1}$ Institute of Hematology "Seràgnoli", University of Bologna, Bologna; ${ }^{2}$ Division of Hematology and Bone Marrow Transplantation, Fondazione IRCCS Istituto Nazionale dei Tumori, University of Milano, Milan; ${ }^{3}$ Dipartimento Biotecnologie Cellulari ed Ematologia, Universitá La Sapienza, Rome; ${ }^{4}$ Department of Medicine and Units of Drug Sciences and Clinical Pharmacology, University Campus Bio-Medico, Rome; ${ }^{5}$ Cardiology Unit, National Cancer Institute IRCCS "Giovanni Paolo II", Bari; ${ }^{6}$ Division of Medical Oncology A, National Cancer Institute, Aviano ; ${ }^{7}$ Center of the Study of Myelofibrosis, Biotechnology Research Area, IRCCS Policlinico S. Matteo Foundation, Pavia; ${ }^{8}$ University of Bologna, Bologna, Italy

\begin{abstract}
Objectives: In this paper, we present a review of critical concepts and research perspectives and produce recommendations on the optimal use of pixantrone in non-Hodgkin lymphoma (NHL) by group discussion from an expert panel appointed by the Italian Society of Hematology and the affiliate societies, Società Italiana di Ematologia Sperimentale and Gruppo Italiano Trapianto di Midollo Osseo. Methods: Recommendations were produced using the Delphi process. Scientific evidence on pixantrone efficacy was analyzed using Grades of Recommendation, Assessment, Development, and Evaluation (GRADE) methodology in the areas where at least one randomized trial was published. The following key issues were addressed for practical recommendations: pixantrone monotherapy in aggressive relapsed or refractory non-Hodgkin B-cell lymphomas and toxicity risk management in patients candidates to pixantrone. Results and conclusions: After a balanced and value-oriented discussion, the panel agreed that the benefit/risk profile was in favor of pixantrone in the treatment of adult patients with multiply relapsed or refractory aggressive NHL B-cell lymphomas. Pixantrone was deemed to be contraindicated in patients with uncontrolled cardiovascular disease. Despite a low rate of cardiotoxicity of pixantrone reported in clinical trials, the panel recommended that all patients receiving pixantrone should undergo periodical cardiac monitoring.
\end{abstract}

Key words pixantrone; guidelines; non-Hodgkin lymphoma; GRADE methodology

Correspondence Giovanni Barosi, MD, Centro per lo Studio della Mielofibrosi, Fondazione IRCCS Policlinico S. Matteo, Viale Golgi 19, 27100, Pavia, Italy. Tel: +39 0382503636; Fax: +39 0382 503917; e-mail: barosig@smatteo.pv.it

Accepted for publication 25 April 2015

doi:10.1111/ejh.12768

Pixantrone is a novel $a z a$-anthracenedione that in preclinical settings showed a remarkable activity in lymphomas and leukemias (1, 2). On May, 2012, the European Medicines Agency (EMA) recommended the granting of a conditional marketing authorization for pixantrone for the treatment of patients with multiply relapsed aggressive NHL as monotherapy $(3,4)$. In the main study submitted for this application, a significant difference in response rate was observed in favor of pixantrone, supported by the results of secondary endpoints of median progression-free (PFS) and overall survival (OS) times (5).
Pixantrone now represents a major advance in the treatment of NHL; however, several uncertainties still remain on the optimal use of the drug in the approved indication, in particular on cardiotoxicity management. In addition, new questions are emerging on the use of the drug early in the treatment of NHL, as well as on its effectiveness as a combination therapy. In order to support the physicians in managing patients candidates to pixantrone, a consensus development conference project on pixantrone was convened under the sponsorship of the Italian Society of Hematology and the affiliate societies Società Italiana di Ematologia 
Sperimentale and Gruppo Italiano Trapianto di Midollo Osseo.

\section{Design and methods}

\section{Organization}

Two chairmen (ST and GB) appointed a panel of seven experts, selected for their expertise in research and clinical practice of adult lymphoid malignancies or in clinical pharmacology, hereafter called expert panel (EP). A clinician with expertise in clinical epidemiology (GB) assured the methodological appropriateness of the process.

\section{GRADE use for evidence appraisal}

We performed a structured literature search for Englishlanguage publications using electronic databases such as MEDLINE (2005-2014), EMBASE (2005-2014), reviews including Cochrane Database of Systematic Reviews, and the Cochrane Controlled Trials Register. References in identified reports and reviews were screened to find additional relevant publications. Publications which measured efficacy of pixantrone in persons with malignant lymphomas with or without a comparison group were included. We used the Grades of Recommendation, Assessment, Development, and Evaluation (GRADE) methodology to rate confidence in estimates of effect for each outcome (6).

\section{The consensus process}

During an initial meeting, the EP agreed on the areas of major concern in the use of pixantrone by generating and rank-ordering clinical key questions using the criterion of clinical relevance, that is, impact on the management of patients and risk of inappropriateness, through a Delphi process (7). The candidate key questions that ranked highest formed the set of issues of the present document. During the first of four meetings, the EP examined the current state of knowledge regarding pixantrone and was accounted for the results of pixantrone critical appraisal. Each panelist drafted statements that addressed the identified key questions, and the remaining panelists scored their agreement with those statements and provided suggestions for rephrasing. For exploiting this phase of the process, the EP was convened and three further consensus meetings were held in Bologna, Italy. The overall goals of the meetings were to reach a definite consensus over question-specific statements for which there was disagreement during the firstround postal phase. The nominal group technique was used by which participants were first asked to comment in round-robin fashion on their preliminary votes and then to propose a new vote (8).

\section{Results}

\section{Preclinical and clinical pharmacology}

Pixantrone differs from mitoxantrone due to removal of the hydroquinone moiety, insertion of a nitrogen heteroatom, and substitution of (ethylamino)-diethylamino for (hydroxyethylamino)-ethylamino side chains. Such chemical modifications cause important effects on pixantrone activity. In preclinical settings, pixantrone showed a limited efficacy in cellular or animal models of solid malignancies but proved remarkably active in lymphomas and leukemias. Pixantrone also showed pharmacodynamics that were different from mitoxantrone. Whereas mitoxantrone killed tumor cells through topoisomerase II $\alpha$ inhibition and consequent formation of DNA double-strand breaks, pixantrone effects on topoisomerase II $\alpha$ were qualitatively similar but quantitatively modest (9).

Pixantrone acts primarily by disrupting mitotic fidelity and segregation of genomic material. This is shown by the formation of lagging chromosomes, chromosomal bridges, micronuclei and multinucleated cells. Apoptosis occurs after 3-4 waves of aberrant mitoses and is accompanied by limited accumulation of DNA double-strand breaks (10). Pixantrone is also able to alkylate DNA and to cause formation of unusually long-lived adducts. This is favored by conjugation of pixantrone with formaldehyde that is formed in measurable amounts in many cancer cells (11). Cause-and-effect relations between persistent DNA damage and topoisomerase II $\alpha$-independent missegregation events are likely to occur; in fact, the mitotic checkpoint inhibitor, pChk1, enhances cellular effects of pixantrone (12).

Pixantrone-induced apoptosis is only in part relayed by p53 (12). This finding anticipates a potential activity of pixantrone in non-Hodgkin lymphomas that harbor loss-of-function p53 mutations $(13,14)$; it also confirms that differences exist between pixantrone and mitoxantrone, whose effects on topoisomerase II $\alpha$ inhibition and DNA damage are relayed to apoptosis by p53 (15). Interestingly, however, recent studies maintain that pixantrone is a topoisomerase II $\alpha$ inhibitor (16). Data scrutiny shows that pixantrone inhibited topoisomerase II $\alpha$ at concentrations exceeding its plasma Cmax in lymphoma patients.

Clinical use of anthracyclines and mitoxantrone is limited by a dose-related cardiotoxicity. Because anthracyclines are eliminated incompletely from cardiac tissue, the risk of cardiotoxicity extends lifetime (17). Sequential exposure to subtoxic doses of different anthracyclines, or sequential exposure to an anthracycline and mitoxantrone, is therefore limited by the risk of precipitating cardiotoxicity. Structural similarities with mitoxantrone and intuitive classification in the large group of anthracycline-like drugs raised concerns that pixantrone caused cardiotoxicity but studies of anthracycline-naïve or anthracycline-pretreated laboratory animals 
soon revealed that pixantrone was much less cardiotoxic than doxorubicin or mitoxantrone and did not aggravate cardiotoxicity in doxorubicin-pretreated animals (18).

Cardiotoxicity from anthracyclines and anthracenediones is a multifactorial event that may be caused by excess formation of reactive oxygen species and other free radicals, or by inhibition of the $\beta$-isoform of topoisomerase II; anthracyclines are also known to convert into long-lived toxic metabolites $(15,19)$. Postcrystallization studies of pixantrone-topoisomerase II $\beta$ complexes, and functional assays (16) suggest that pixantrone would not target cardiac topoisomerase II $\beta$. In a translational model of human heart pre-exposed to doxorubicin, pixantrone did not produce free radicals but actually inhibited formation of the long-lived and toxic doxorubicin metabolite, doxorubicinol. In comparable settings, mitoxantrone did produce free radicals and lacked inhibition of doxorubicinol formation (20). Pixantrone generation of reactive oxygen species only occurs in cell free systems that adopt high drug concentrations (16).

All such findings provide additional evidence to conclude that pixantrone is different from mitoxantrone and anthracyclines.

\section{Recommendations}

\section{Pixantrone monotherapy in aggressive relapsed or refractory non-Hodgkin B-cell lymphomas}

Early phase I or phase II trials with pixantrone. The standard approach for adult patients with aggressive NHL not achieving a complete remission or relapsing after anthracycline-based induction and eligible for intensive treatment includes a salvage combination based on the anti-CD20 antibody rituximab (in CD20-positive NHL) and a noncross-resistant platinum-containing regimen. The most used regimens are DHAP/DHAOX (dexamethasone, cytarabine, and cisplatin/oxaliplatin), ICE (ifosfamide, carboplatin, etoposide), or ESHAP (methylprednisolone, etoposide, cytarabine, cisplatin). Responders to re-induction usually undergo consolidation with high-dose therapy and autologous stem cell transplantation (ASCT); in selected cases, allogeneic stem cell transplantation (allo-SCT) may be considered. In the planning of treatment for patients in this setting, one of the trickiest issues that physicians have to face is progressive myocardial toxicity related to the cumulative, dose-dependent damage induced by anthracyclines, which may lead to congestive heart failure (CHF).

Pixantrone was synthesized to provide similar antitumor activity similar to the anthracyclines, but without the cardiotoxicity. Three phase I dose-escalation single-agent studies (two in solid tumors and one in NHL/chronic lymphocytic leukemia) explored two different treatment regimens with pixantrone monotherapy: pixantrone every 3 wk and pixantrone weekly for three consecutive weeks with 1-week rest (21-23). The final schedule selected for phase II development was $85 \mathrm{mg} / \mathrm{m}^{2}$ of pixantrone on days 1,8 , and 15 of a 28 -d cycle.

Following the promising results of pixantrone in earlier trials, pixantrone has been tested as a single agent in a prospective phase II study (24). Five patients achieved a complete remission (CR) (15\%) and nine had a partial response (PR) (27\%), with a median PFS of $106 \mathrm{~d}$. Overall response rate (ORR) was higher than that reported for single-agent etoposide, cisplatin, or mitoxantrone.

Phase III randomized trial comparing pixantrone with single-agent chemotherapy. The only phase III trial was an international, multicenter, randomized, active-controlled, open-label study reported by Pettengell and coworkers (PIX 301) and it led to drug registration by the EMA (5). The study recruited patients with relapsed/refractory aggressive or transformed NHL refractory to at least two prior regimens, including at least one anthracycline-containing regimen (median cumulative dose of approximately $300 \mathrm{mg}$ of doxorubicin equivalents $/ \mathrm{m}^{2}$ ). Patients were randomized $(1: 1)$ to receive either pixantrone (with dose and schedule identical to that used in the phase II setting) or single-agent chemotherapy of the investigators choice, some of them being: vinorelbine, ifosfamide, oxaliplatin, etoposide, gemcitabine, or mitoxantrone. The primary endpoints were CR and complete remission unconfirmed $(\mathrm{CRu})$ with PFS and OS as secondary endpoints. The initial planned sample size was 320 patients, but the study was subsequently closed $3 \mathrm{yr}$ after the first patient was enrolled, with only 140 patients randomized (70 per arm) due to very slow accrual. Most of the enrolled patients had diffuse large B-cell lymphoma $(76 \%$ in the pixantrone arm vs. $73 \%$ in comparator arm) or transformed indolent lymphoma (14\% vs. 13\%), with a median age of $60 \mathrm{yr}$ vs. $58 \mathrm{yr}$, mainly unfavorable Ann Arbor stage and International Prognostic Index (IPI) scores.

Patients living in countries where rituximab was available were only eligible if they had received rituximab therapy. A similar number of patients in each group had previously received rituximab. Baseline patient characteristics were well balanced in experimental and control arms. Seventy-one percent of patients in the experimental arm and $76 \%$ in the comparator arm did not complete the six planned cycles because of disease progression or relapse $(41 \%$ in the pixantrone group vs. $58 \%$ in the comparator group) or adverse events (AEs) (22\% vs. $13 \%$, respectively). The response analysis, based on the intention-to-treat population, showed a benefit in terms of $\mathrm{CR} / \mathrm{CRu}$ rate and ORR for pixantrone (20\% vs. $5.7 \%, P=0.021 ; 37.1 \%$ vs. $14.3 \%, P=0.003$, respectively). Median PFS was longer in the experimental arm (5.3 months vs. 2.6 months, $P=0.005$ ), and a trend toward longer median OS was observed with pixantrone, but this was not statistically significant (10.2 months vs. 7.6 months, $P=0.251)$. An exploratory analysis was 
performed to investigate whether any favorable factor predicting a better outcome was recognizable; an absence of prior anti-CD20 treatment or stem cell transplantation, less than three prior chemotherapy regimens, age $\geq 65 \mathrm{yr}$, and female sex were identified as favorable prognostic factors, but prior use of rituximab did not influence the benefit on PFS.

In terms of toxicity, patients in the experimental arm experienced more grade III and IV AEs $(76.5 \%$ vs. $52.2 \%$ ); however, the overall proportion of complications was similar in the two groups $(97.1 \%$ vs. $91 \%)$. No evidence of cumulative, dose-related cardiotoxicity was reported, and decreases in left ventricular ejection fraction (LVEF) were not associated with clinical evidence of cardiac impairment.

Critical appraisal of evidence and panel discussion on appropriateness of pixantrone in advanced NHL. A critical appraisal of evidence resulting from the randomized openlabel controlled clinical trial according to GRADE methodology (6) is reported in the Supplement material. Two main reasons for downgrading the quality of evidence supporting the use of pixantrone in relapsed or refractory NHL were highlighted. These were imprecision of the estimate of OS and indirectness of the population treated in the trial. As a matter of fact, the difference in OS between experimental arm and control arm was not statistically significant. Consequently, responders would not be judged responders using OS. This discordance, reflecting indirectness of the OS outcome measurement in the trial, could influence the confidence in the effect size measurement because a proportion of the responses were not clinically meaningful. Moreover, the EMA approval of pixantrone did not specify an exclusion for patients having previously received rituximab. Thus, entry criteria of the trial differed substantially from the EMA-approved therapy indication. Because of these two issues, the risk of indirectness in the population being considered for therapy is high.

These conclusions were provided to the EP for discussion. As far as imprecision in the estimate of OS, the EP reasoned that the study was powered to look at response rate (RR) and PFS (primary endpoints) and that, in the setting of patients with relapsed/refractory NHL, RR and PFS were deemed appropriate quality indicator of outcome. Thus, PFS may well be intended as a surrogate for OS. Moreover, the EP claimed that the real role of pixantrone therapy in multiply relapsed refractory NHL patients is the potential 'bridge' to SCT, rendering OS outcome not so critical for these patients in which the principal aim is to obtain a clinical response (CR-CRu) to lead the patient (if eligible) to a consolidation SCT. In the EP's opinion, the low precision level in the estimate of OS for pixantrone superiority over investigator's choice in rituximab-treated patients neither affects credibility of findings in PIX 301 study nor introduces concerns in using pixantrone as per EMA recommendation.
As far as the indirectness of the population, the EP agreed that the inclusion of rituximab-naive patients in the study represented a bias in the quality of evidence of pixantrone activity. In fact, nowadays almost all patients would be expected to have received previous rituximab, and previous exposure to this antibody is known to be associated with a decreased RR to subsequent chemotherapy. However, the EP highlighted the evidence of poor outcome in the analysis of patients naïve and non-naïve to rituximab derived from a substudy, resulting in a very limited number of patients. Despite this small number of patients, the RR in those previously exposed to rituximab was 1.76 (95\% CI, 0.77-3.98). This was not statistically significant but considered by the EP as clinically relevant. The EP agreed that the results in patients naive to rituximab do not affect the overall credibility of findings in Pettengell's study. The EP also acknowledged that cost-effectiveness of pixantrone was positively evaluated by NICE and others $(25,26)$.

Recommendations. According to EMA, pixantrone is indicated for the treatment of adult patients with multiply relapsed or refractory aggressive NHL B-cell lymphomas.

The EMA approval was based on only one randomized trial whose evidence of the better benefit of pixantrone with respect to available therapies was judged moderate. In particular, the evidence on the risk/benefit risk profile of the drug was judged of lower quality in patients who had received a previous rituximab therapy.

After a balanced and value-oriented discussion, the panel agreed that the benefit/risk profile was in favor of pixantrone in any category of patients despite the previous treatment (recommendation of moderate strength).

\section{Toxicity risk management in patients candidates to pixantrone}

During treatment with pixantrone as single agent in relapsed aggressive NHL, grade 3-4 leukopenia and neutropenia were the most relevant hematological toxicity $(5,24)$. These were generally brief, lasting a median of $7.5 \mathrm{~d}$, and did not increase with the number of cycles, whereas grade 3-4 anemia or thrombocytopenia was rarely observed. In particular, in the Pettengels's trial (5), grade III-IV neutropenia was more common in patients treated with pixantrone (41.2\% vs. $19.4 \%)$, as was febrile neutropenia $(7.4 \%$ vs. $3.0 \%)$, while the rate of thrombocytopenia was similar and that of anemia was lower ( $11.8 \%$ vs. $10.4 \%$ and $5.9 \%$ vs. $13.4 \%$, respectively).

In the open-label, non-randomized, non-comparative phase II study (24), only three patients, two of whom with a previous low value of (LVEF), showed a $\geq 10 \%$ decrease in LVEF measured by multigated acquisition scan. In one of these, the treatment had to be discontinued when the patient developed cardiac symptoms and LVEF decreased to $25 \%$. All of patients with cardiovascular toxicity were more than $65 \mathrm{yr}$ of age and had received a prior anthracycline. 
In the pivotal phase III trial (5), cardiac toxicity was closely monitored, and a higher incidence of cardiac events was seen in the pixantrone group (35\% vs. $21 \%$ ). Only $13 \%$ cases of cardiac events were considered related to pixantrone, and all were asymptomatic decreases in LVEF. No clear cases of pixantrone-associated CHF were reported, and importantly, cardiac events did not correlate with the cumulative dose of pixantrone. This latter finding denoted differences between pixantrone and anthracyclines, whose cardiotoxicity typically develops in a dose-related manner. Five patients in the experimental arm had a previous history of $\mathrm{CHF}$ or continuing cardiomyopathy at the time of enrollment, whereas none of the patients in the comparator group had a history of these conditions. Such an unbalanced representation of cardiac risk factors in the two study arms could have at least in part contributed to the observed higher incidence of cardiac adverse events in patients randomized to pixantrone. Overall, pixantrone cardiotoxicity is judged to be acceptable, especially if one appreciates that patients had been previously exposed to a median cumulative doxorubicin dose of approximately $300 \mathrm{mg} / \mathrm{m}^{2}$. The acceptable cardiotoxicity of pixantrone is confirmed by the Periodic Safety Report (PSUR) that summarizes pixantrone safety data from both clinical and commercial data, the vast majority of cardiac events attributed to pixantrone being coded 'investigations' (ejection fraction decreased) rather than cardiac disorders (27).

Clinical and health conditions more frequently related to cardiovascular events during pixantrone administration are the age greater than $65 \mathrm{yr}$, the low LVEF value before therapy, the previous history of CHF, and cardiomyopathy.

No evidence was reported for the efficacy of cardiovascular prophylaxis in patients candidates to pixantrone and carrying these risk factors. No recommendations for prophylaxis or treatment of cardiac toxicity were reported.

\section{Recommendations}

The EP agreed that neutropenia ensuing after pixantrone single-agent therapy should be managed by G-CSF prophylaxis as for other NHL chemotherapy regimens (28).

Pixantrone is contraindicated in patients with uncontrolled cardiovascular disease.

All patients without cardiovascular disease or with a controlled cardiovascular disease who are candidates for pixantrone therapy should undergo a cardiological evaluation including electrocardiogram and echocardiogram. There are no indications to cardiovascular prophylaxis in patients without cardiovascular disease.

In patients on treatment for cardiovascular disease, the appropriateness of cardiovascular therapy should be verified before pixantrone therapy.

Despite a low rate of cardiotoxicity of pixantrone reported in clinical trials, the EP recommended that all patients receiving pixantrone should undergo periodical cardiac monitoring.

If cardiac toxicity is demonstrated during treatment (grade two cardiac toxicity (29) or significant decrease in LVEF), the individual risk/benefit ratio of continuing pixantrone therapy should be evaluated.

\section{Research perspectives}

\section{Pixantrone combination therapy in relapsed or refractory non-Hodgkin B-cell lymphomas}

Four studies have investigated pixantrone as part of a polychemotherapeutic regimen, in patients with relapsed NHL. Three studies included patients with aggressive NHL, and one study included patients with indolent NHL. Three studies were reported in full papers while one in abstract form (30-33) (Table 1). Taken overall, the responses ranged from $58 \%$ to $73 \%$. Cardiotoxicity rates ranged from $7 \%$ to $19 \%$ with limited grade II-IV toxicities (2-3\%). These results

Table 1 Studies using pixantrone in combination therapy for patients with refractory or resistant aggressive NHL

\begin{tabular}{|c|c|c|c|c|c|c|c|c|c|}
\hline Study & Design & Combination & $\begin{array}{l}\text { Number of } \\
\text { patients } \\
\text { treated }\end{array}$ & $\begin{array}{l}\text { Media number } \\
\text { of cycles } \\
\text { administered }\end{array}$ & $\begin{array}{l}\text { ORR, } \\
n(\%)\end{array}$ & $\begin{array}{l}\mathrm{CR} \\
n(\%)\end{array}$ & $\begin{array}{l}\mathrm{PR} \\
n(\%)\end{array}$ & $\begin{array}{l}\text { Median } \\
\text { PFS }\end{array}$ & OS \\
\hline $\begin{array}{l}\text { Lim et al., } \\
(2007)(30)\end{array}$ & Phase I/II & PSHAP & 19 & $4(1-6)$ & $11(58)$ & 7 (37) & $4(21)$ & 5.7 months & \multirow{3}{*}{$\begin{array}{l}\text { Median: } \\
14.7 \text { months } \\
\text { Median: } \\
17.9 \text { months }\end{array}$} \\
\hline $\begin{array}{l}\text { Borchman et al., } \\
\text { (2011) (31) }\end{array}$ & Phase I/II & CPOP & 65 & $6(1-6)$ & $47(72)$ & $30(46)$ & $17(26)$ & 8.2 months & \\
\hline $\begin{array}{l}\text { d'Amore et al., } \\
\text { (2014) (32) }\end{array}$ & $\begin{array}{l}\text { Preliminary } \\
\text { clinical } \\
\text { experience }\end{array}$ & $\begin{array}{l}\text { PREBEN/ } \\
\text { PEBEN }\end{array}$ & 8 & $3(2-6)$ & $6(62.5)$ & $3(37.5)$ & $2(25)$ & & \\
\hline $\begin{array}{l}\text { Srokowski et al., } \\
\text { (2011) (33) }\end{array}$ & $\begin{array}{c}\text { Dose-escalation } \\
\text { phase I study }\end{array}$ & FPD-R & 27 & $5(1-8)$ & $19(70)$ & $12(44)$ & $7(26)$ & & $\begin{array}{c}2 \text { and } \\
\text { 3-yr: } 91.5 \%\end{array}$ \\
\hline
\end{tabular}

PSHAP, pixantrone, methylprednisolone, high-dose cytarabine, cisplatin; CPOP, cyclophosphamide, pixantrone, vincristine, prednisone; FPD-R, fludarabine, pixantrone, dexamethasone, rituximab; PREBEN/PEBEN, etoposide, bendamustine, pixantrone ( \pm rituximab). 
suggest that pixantrone can be further evaluated in combination studies.

Ideal candidates for combination therapy with pixantrone in NHL should target molecular pathways other than those of pixantrone or should be able to remove molecular barriers to the action of pixantrone. Combination agents and pixantrone would therefore elicit synergistic rather than additive effects in tumor cells. The finding that clinically relevant concentrations of pixantrone lacked inhibition of topoisomerase II $\alpha$ anticipates that non-anthracycline topoisomerase II $\alpha$ inhibitors would synergize with pixantrone in tumor cells. The epipodophyllotoxin, etoposide, is a general topoisomerase inhibitor that should be considered for combination with pixantrone. Other combination strategies might adopt inhibitors of mitotic checkpoints. The mixed antimetabolite alkylator, bendamustine, warrants consideration as it induces a complex stress response that downregulates the activity of numerous mitotic checkpoints (34).

Among the combination therapies including pixantrone, only one trial fitted this rationale. d'Amore and coworkers (32) explored a combination chemotherapy regimen based on pixantrone in patients with multiply relapsed or refractory aggressive B and T non-Hodgkin lymphoma in which etoposide and bendamustine were chosen as companion compounds. Rituximab was added in patients with B-cell lymphoma. The adapted schedule consisted of pixantrone $50 \mathrm{mg} / \mathrm{m}^{2}$ i.v. day $1+8$, etoposide $100 \mathrm{mg}$ i.v. day 1 , bendamustine $90 \mathrm{mg} / \mathrm{m}^{2}$ i.v. day 1 with or without the addition of rituximab $375 \mathrm{mg} / \mathrm{m} 2$ i.v. day 1 (PREBEN/PEBEN). If feasible, each cycle was given at 3-weekly intervals for a maximum of six cycles. Three patients $(25 \%)$ achieved a CR and $2(50 \%)$ obtained a partial response (ORR 62.5\%). One trial with ongoing enrollment is registered on ClinicalTrials.gov (NCT 01491841) aimed at testing bendamustine, rituximab, and pixantrone in a phase I/II trial in patients with relapsed/refractory B-cell NHL.

Two other relevant trials are registered on the ClinicalTrials.gov website, both concerning the treatment of relapsed refractory B-cell NHL. The first study investigates relapsed diffuse large B-cell lymphoma, transformed from indolent lymphoma, and follicular grade III lymphoma not eligible for SCT. This phase III, multicenter, randomized trial is comparing a pixantrone-rituximab regimen with a gemcitabine-rituximab regimen, with a planned sample size of 350 patients (NCT01321541). The second one was aimed to evaluate the ORR of obinutuzumab (GA101) in combination with pixantrone. Seventy patients will receive up to six cycles of the regimen. (NCT02499003).

\section{Pixantrone combination therapy in untreated DLBC Iymphoma}

Herbrecht and coworkers initiated a phase II multicenter, randomized controlled trial in untreated patients with stage
II-IV DLBCL substituting doxorubicin with pixantrone $\left(150 \mathrm{mg} / \mathrm{m}^{2}\right)$ in R-CHOP (R-CPOP) (35). After four cycles, patients with a $(\mathrm{PR})$ received four more cycles of treatment; those with $\mathrm{CR}$ received two more cycles. The primary objective of the trial was to show non-inferiority of R-CPOP to R-CHOP, and secondary endpoints included ORR, PFS, OS, and safety. The study recruited 124 from the preplanned 280 patients needed, due to financial constraints. Of the 124 patients enrolled, 61 were randomized to R-CPOP and 63 to R-CHOP. In terms of efficacy, there were no statistically significant differences between the two regimens. $\mathrm{CR}$ rate in the intent-to-treat population was $72.1 \%$ for R-CPOP vs. $79.4 \%$ for R-CHOP, and ORR was $82 \%$ vs. $87.3 \%$. Median PFS was not reached in the CPOP-R arm and was 40 months in the CHOP-R arm; median OS was not reached in either treatment arm. OS rates were lower for patients treated with CPOP-R (hazard ratio 2.37, $P=0.029$ ), with more deaths occurring in the CPOP-R arm (30\% vs. $14 \%)$. Overall, AEs were similar in approximately $85 \%$ of patients in both arms. Non-inferiority could not be demonstrated due to early closure of the trial due to financial constraints. Despite this, efficacy was comparable between the two arms, but R-CPOP showed a reduced cardiotoxicity measured by echocardiography or circulating levels of troponin.

\section{References}

1. Boyle EM, Morschhauser F. Pixantrone: a novel anthracycline-like drug for the treatment of non-Hodgkin lymphoma. Expert Opin Drug Saf 2015;14:601-7.

2. Mukherji D, Pettengell R. Pixantrone for the treatment of aggressive non-Hodgkin lymphoma. Expert Opin Pharmacother 2010;11:1915-23.

3. Péan E, Flores B, Hudson I, Sjöberg J, Dunder K, Salmonson T, Gisselbrecht C, Laane E, Pignatti F. The European Medicines Agency review of pixantrone for the treatment of adult patients with multiply relapsed or refractory aggressive nonHodgkin's B-cell lymphomas: summary of the scientific assessment of the committee for medicinal products for human use. Oncologist 2013;18:625-33.

4. Pettengell R, Kaur J. Pixantrone dimaleate for treating nonHodgkin's lymphoma. Expert Opin Orphan Drugs 2015;3:747-57.

5. Pettengell R, Coiffier B, Narayanan G, et al. Pixantrone dimaleate versus other chemotherapeutic agents as a singleagent salvage treatment in patients with relapsed or refractory aggressive non-Hodgkin lymphoma: a phase 3, multicentre, open-label, randomised trial. Lancet Oncol 2012;13:696-706.

6. Guyatt GH, Oxman AD, Vist GE, Kunz R, Falck-Ytter Y, Alonso-Coello P, Schünemann HJ; GRADE Working Group. GRADE: an emerging consensus on rating quality of evidence and strength of recommendations. BMJ 2008;336:924-6.

7. Delbecq AL, van de Ven AH, Gustafson DH. Group Techniques for Program Planning: A guide to nominal group and 
Delphi processes. Scott, Foresman and Co, Glenview: IL, USA; 1975.

8. William PL, Webb C. The Delphi technique: a methodological discussion. J Adv Nurs 1994;19:180-6.

9. De Isabella P, Palumbo M, Sissi C, et al. Topoisomerase II DNA cleavage stimulation, DNA binding activity, cytotoxicity, and physico-chemical properties of 2-aza- and 2-azaoxide-anthracenedione derivatives. Mol Pharmacol 1995;48:30-8.

10. Beeharry N, Di Rora AG, Smith MR, Yen TJ. Pixantrone induces cell death through mitotic perturbations and subsequent aberrant cell divisions. Cancer Biol Ther 2015;16:1397-406.

11. Mansour OC, Evison BJ, Sleebs BE, et al. New anthracenedione derivatives with improved biological activity by virtue of stable drug-DNA adduct formation. $J$ Med Chem 2010;53:6851-66.

12. Beeharry N, Zhu X, Murali V, Smith MR, Yen T. Pixantrone induces cell death through mitotic perturbations and subsequent aberrant cell divisions in solid tumor cell lines. Mol Cancer Ther 2013;12(11 Suppl):A147.

13. Møller MB, Gerdes AM, Skjødt K, Mortensen LS, Pedersen NT. Disrupted p53 function as predictor of treatment failure and poor prognosis in B- and T-cell non-Hodgkin's lymphoma. Clin Cancer Res 1999;5:1085-91.

14. Wilson WH, Teruya-Feldstein J, Fest T, Harris C, Steinberg SM, Jaffe ES, Raffeld M. Relationship of p53, bcl-2, and tumor proliferation to clinical drug resistance in non-Hodgkin's lymphomas. Blood 1997;89:601-9.

15. Minotti G, Menna P, Salvatorelli E, Cairo G, Gianni L. Anthracyclines: molecular advances and pharmacologic developments in antitumor activity and cardiotoxicity. Pharmacol Rev 2004;56:185-229.

16. Hasinoff BB, Wu X, Patel D, Kanagasabai R, Karmahapatra $\mathrm{S}$, Yalowich JC. Mechanisms of action and reduced cardiotoxicity of pixantrone; a topoisomerase ii targeting agent with cellular selectivity for the topoisomerase ii $\alpha$ isoform. $J$ Pharmacol Exp Ther 2016;356:397-409.

17. Minotti G, Salvatorelli E, Menna P. Pharmacological foundations of cardio-oncology. J Pharmacol Exp Ther 2010;334:2-8.

18. Cavalletti E, Crippa L, Mainardi P, Oggioni N, Cavagnoli R, Bellini O, Sala F. Pixantrone (BBR 2778) has reduced cardiotoxic potential in mice pretreated with doxorubicin: comparative studies against doxorubicin and mitoxantrone. Invest New Drugs 2007;25:187-95.

19. Zhang S, Liu X, Bawa-Khalfe T, Lu LS, Lyu YL, Liu LF, Yeh ET. Identification of the molecular basis of doxorubicininduced cardiotoxicity. Nat Med 2012;18:1639-42.

20. Salvatorelli E, Menna P, Paz OG, Chello M, Covino E, Singer JW, Minotti G. The novel anthracenedione, pixantrone, lacks redox activity and inhibits doxorubicinol formation in human myocardium: insight to explain the cardiac safety of pixantrone in doxorubicin-treated patients. J Pharmacol Exp Ther 2013;344:467-78.

21. Borchmann P, Schnell R, Knippertz R, et al. Phase I study of BBR 2778, a new aza-anthracenedione, in advanced or refractory non-Hodgkin's lymphoma. Ann Oncol 2001;12:661-7.

22. Faivre S, Raymond E, Boige V, Gatineau M, Buthaut X, Rixe O, Bernareggi A, Camboni G, Armand JP. A phase I and pharmacokinetic study of the novel aza-anthracenedione compound BBR 2778 in patients with advanced solid malignancies. Clin Cancer Res 2001;7:43-50.

23. Dawson LK, Jodrell DI, Bowman A, Rye R, Byrne B, Bernareggi A, Camboni G, Smyth JF. A clinical phase I and pharmacokinetic study of BBR 2778, a novel anthracenedione analogue, administered intravenously, 3 weekly. Eur J Cancer 2000;36:2353-9.

24. Borchmann P, Morschhauser F, Parry A, et al. Phase-II study of the new aza-anthracenedione, BBR 2778, in patients with relapsed aggressive non-Hodgkin's lymphomas. Haematologica 2003;88:888-94.

25. National Institute for Health and Care Excellence. Pixantrone monotherapy for treating multiply relapsed or refractory aggressive non-Hodgkin's B cell lymphoma. NICE technology appraisal guidance [TA306]. Published date: February 2014. http://www.nice.org.uk/guidance/TA306

26. Muszbek N, Kadambi A, Lanitis T, Hatswell AJ, Patel D, Wang L, Singer JW, Pettengell R. The Cost-effectiveness of Pixantrone for third-/fourth-line treatment of aggressive nonHodgkin's Lymphoma. Clin Ther. 2016;38:503-15.

27. CTI Biopharma Periodic Safety Update Report \#6 (10 November 2014 to 09 May 2015). Report date 13 July 2015

28. Flowers CR, Seidenfeld J, Bow EJ, et al. Antimicrobial prophylaxis and outpatient management of fever and neutropenia in adults treated for malignancy: American Society of Clinical Oncology clinical practice guideline. J Clin Oncol 2013;31:794-810.

29. Common Terminology Criteria for Adverse Events (CTCAE) Version 4.0s Published: May 28, 2009 (v4.03: June 14, 2010) http://evs.nci.nih.gov/ftp1/CTCAE/CTCAE_4.03_2010-0614_QuickReference_5x7.pdf. (Accessed 7 January 2016)

30. Lim ST, Fayad L, Tulpule A, Modiano M, Cabanillas F, Laffranchi B, Allievi C, Bernareggi A, Levine AM. A phase I/II trial of pixantrone (BBR2778), methylprednisolone, cisplatin, and cytosine arabinoside (PSHAP) in relapsed/refractory aggressive non-Hodgkin's lymphoma. Leuk Lymphoma 2007;48:374-80.

31. Borchmann P, Herbrecht R, Wilhelm M, Morschhauser F, Hess G, Cernohous P, Veals SA, Singer JW, Engert A. Phase I/II study of pixantrone in combination with cyclophosphamide, vincristine, and prednisone in patients with relapsed aggressive non-Hodgkin lymphoma. Leuk Lymphoma 2011;52:620-8.

32. d'Amore F, Joergensen J, Sillesen IB, et al. Preliminary clinical experience on the efficacy and feasibility of a new combination regimen consisting of pixantrone, etoposide, and bendamustine with or without the addition of rituximab in patients with relapsed/refractory aggressive non-Hodgkin lymphomas. Blood 2014; 124:abstract 5435.

33. Srokowski TP, Liebmann JE, Modiano MR, Cohen GI, Pro B, Romaguera JE, Kuepfer C, Singer JW, Fayad LE. 
Pixantrone dimaleate in combination with fludarabine, dexamethasone, and rituximab in patients with relapsed or refractory indolent non-Hodgkin lymphoma: phase 1 study with a doseexpansion cohort. Cancer 2011;117:5067-73.

34. Leoni LM, Bailey B, Reifert J, Bendall HH, Zeller RW, Corbeil J, Elliott G, Niemeyer CC. Bendamustine (Treanda) displays a distinct pattern of cytotoxicity and unique mechanistic features compared with other alkylating agents. Clin Cancer Res 2008;14:309-17.

35. Herbrecht R, Cernohous P, Engert A, et al. Comparison of pixantrone-based regimen (CPOP-R) with doxorubicin-based therapy (CHOP-R) for treatment of diffuse large B-cell lymphoma. Ann Oncol 2013;24:2618-23. 